REVUE DE L'INSTITUT

FRANÇAIS D'HISTOIRE

EN ALLEMAGNE

\section{Revue de I'IFHA}

Revue de l'Institut français d'histoire en Allemagne

$1 \mid 2009$

IFHA 1

\title{
Charlemagne et les « objets ». Des thésaurisations carolingiennes aux constructions mémorielles
}

Journée d'études, Paris-Auxerre, 8-10 octobre 2008

\section{Marianne Besseyre et Philippe Cordez}

\section{OpenEdition}

\section{Journals}

Édition électronique

URL : http://journals.openedition.org/ifha/314

DOI : $10.4000 /$ ifha. 314

ISSN : 2198-8943

Éditeur

IFRA - Institut franco-allemand (sciences historiques et sociales)

Édition imprimée

Date de publication : 30 septembre 2009

Pagination : 26-31

ISSN : 2190-0078

Référence électronique

Marianne Besseyre et Philippe Cordez, «Charlemagne et les « objets ». Des thésaurisations carolingiennes aux constructions mémorielles », Revue de l'IFHA [En ligne], 1 | 2009, mis en ligne le 07 février 2013, consulté le 15 septembre 2020. URL : http://journals.openedition.org/ifha/314

Ce document a été généré automatiquement le 15 septembre 2020

(CIFHA 


\section{Charlemagne et les « objets ». Des thésaurisations carolingiennes aux constructions mémorielles}

Journée d'études, Paris-Auxerre, 8-10 octobre 2008

Marianne Besseyre et Philippe Cordez

\section{NOTE DE L'ÉDITEUR}

Rapport établi par Marianne Besseyre et Philippe Cordez

Ces journées consacrées à Charlemagne et à ses "objets », qui débutèrent par une rencontre sur le terrain dans plusieurs institutions patrimoniales parisiennes avant de se poursuivre au Centre d'études médiévales d'Auxerre entre le 8 et le 10 octobre 2008, s'enracinaient dans plusieurs projets antérieurs. D'une part, des réflexions menées depuis plusieurs années par un groupe informel d'historiens et d'historiens de l'art à propos de la notion et de la pratique du " trésor » au Moyen Âge avaient pu s'affûter en 2006 à l'occasion d'un premier colloque ${ }^{1}$, en marge duquel il était apparu que la figure tant historique que mémorielle de Charlemagne avait catalysé, comme aucune autre, l'invention de nouvelles pratiques et de nouveaux types d'« objets » : il était tentant de prolonger cette enquête collective autour du souverain carolingien. D'autre part, l'exposition Trésors carolingiens. Livres manuscrits de Charlemagne à Charles le Chauve, organisée à la Bibliothèque Nationale de France en $2007^{2}$, avait porté l'attention sur un ensemble de manuscrits qui, par leur ambition politique et leur préciosité fascinante, invitaient à approcher le phénomène des «objets-livres» en interrogeant parallèlement leurs évolutions formelles et leurs transformations conceptuelles.

Les artefacts transmis par le passé sont, pour les historien(ne)s, les « objets » d'amours secrètes que le musée révèle, ils sont un " pain quotidien ", ou encore, pour prendre au mot la métaphore banalisée par l'Académie, ils sont les « sources » où doit s'étancher leur « soif ». D'où la conviction que cette question des « objets » est à la base du métier, 
et qu'elle doit être approchée historiquement, comme notion et comme pratique. Il s'agit d'analyser des discours et des contextes humains, tout en exploitant le potentiel herméneutique de notre appréhension sensible de la culture matérielle d'une époque : c'est là que résidaient, à expérimenter au prétexte de Charlemagne, les enjeux de la rencontre. Or une telle mobilisation conjointe des aptitudes sensibles et abstraites du sujet historien devant ses "objets", qui exige des circonstances favorables pour s'épanouir, reste trop souvent un enjeu d'abord institutionnel et pragmatique, tant les différentes «cultures de l'objet» sont à l'origine, parmi les acteurs du milieu scientifique eux-mêmes, de variations subjectives qui accèdent mal au niveau de la conscience et demeurent des obstacles, au lieu d'être fertiles.

La manifestation commença donc par une confrontation physique avec des « objets de Charlemagne » - c'est-à-dire lui ayant appartenu dans les faits ou dans la légende - en mettant à profit le fait, lié à l'histoire de la France, que la ville de Paris est celle qui en réunit la plus grande concentration. Un petit groupe de taille variable, où l'on intervenait à tour de rôle en fonction de sa connaissance des objets, pérégrina donc le temps d'un après-midi sur les "traces" de Charlemagne. Le départ fut donné au Cabinet des Médailles et au Département des manuscrits de la Bibliothèque nationale de France, où quelques collègues s'étaient éclipsés de l'Institut d'Histoire de l'Art voisin pour nous rejoindre, et où tous furent aisément convaincus des vertus cognitives de la manipulation des objets, démontrée par Marianne Besseyre (conservateur au Centre de Recherche sur les Manuscrits Enluminés). On se transporta ensuite au Musée National du Moyen Âge, où Isabelle Bardiès-Fronty (conservateur responsable des collections du haut Moyen Âge) présenta certains objets du musée en insistant particulièrement sur l'importance artistique et historique du foyer messin depuis Pépin le Bref, le grand aïeul dynastique, puis au Département des Objets d'Art du Musée du Louvre, où Yann Potin (Université de Paris X Nanterre) intervint sur la mémoire carolingienne des rois de France. L'équipée fut joyeuse, même si son rythme fut rapide ; elle permit de nouer des liens qui allaient être profitables aux échanges poursuivis les jours suivants, dans le cadre accueillant de la maison du Coche d'eau à Auxerre, où l'on revint plus d'une fois sur certains objets vus à Paris.

La première partie des conférences, qui portait sur les «thésaurisations carolingiennes", fut introduite par Claire Sonnefraud (ENSSIB, Lyon: "La réforme carolingienne et les "inventaires de trésors " ") à propos des inventaires d'objets mobiliers ecclésiastiques carolingiens. Leur rédaction et leur tradition témoignent du rôle de l'écrit dans l'entreprise réformatrice, que l'on reconnaît aussi dans les initiatives législatives contemporaines, ainsi que d'une volonté conjointe de mettre en place une image de "renaissance ", afin de la léguer à la postérité. Mark Mersiowsky (Universität Stuttgart / Monumenta Germaniae Historica, Munich : « Entre preuve et objet sacré. Diplômes et chartes carolingiens et leurs fonctions contemporaines ») explorait ensuite les circonstances de la rédaction et de la conservation des documents délivrés par les souverains carolingiens, démontrant leur activité permanente de confirmation et de célébration des liens politiques constitutifs du nouvel ordre carolingien.

Les deux contributions suivantes portèrent sur l'enluminure : observant l'évolution des modes de représentation des « objets » à la fin du VIIIe et au cours du IXe siècle, Daniel Russo (Université de Bourgogne, Dijon: «Plans, fonds, surfaces : définition de l'objet dans l'aire visuelle à l'époque carolingienne ») mit en évidence l'affirmation croissante de la présence sensible des artefacts, matériels ou figurés; elle s'exprime par l'emploi de matières précieuses ou par une construction spatiale de l'imago qui creuse l'espace 
de façon toujours plus signifiante, densifiant et réifiant l'objet livre de l'intérieur comme à l'extérieur, pour faire finalement du manuscrit dans son ensemble un « objet de trésor ", pesant de tout son poids politique et sacré. Approchant ce même phénomène en reprenant le dossier énigmatique d'un sacramentaire particulièrement somptueux, mais resté inachevé, Marianne Besseyre (BnF, Manuscrits, CRME, Paris : «Un fragment de Parole orfévré : le Sacramentaire dit de Charles le Chauve (BnF, ms. lat. 1141) et la question du « livre-relique » ») s'interrogeait sur la thésaurisation des inventions formelles d'un bout à l'autre de l'époque carolingienne et sur ses enjeux mémoriels - l'emblème de cette problématique résidant dans la mystérieuse identité du « portrait cumulé » du souverain contemporain figuré dans le Sacramentaire, au regard de ses modèles prestigieux, Charlemagne ou Constantin.

$\mathrm{Au}$ terme de cette première section, le constat s'imposait que le moment carolingien vit l'épanouissement d'une " politique des objets » d'un nouveau genre, aussi bien pour la production écrite que dans la création d'objets précieux: celle-ci fut initiée par Charlemagne (768-814) et rendue possible par la mobilisation d'un vaste réseau de compétences au sein d'une structure hiérarchique capitalisant les expériences; elle s'amplifia à travers le prisme mémoriel du premier empereur. Telle était la situation à la fin du règne de Charles le Chauve (843-877), qui coïncide avec l'apogée artistique carolingien : restait à observer comment cet héritage singulier et complexe, doté d'une grande cohérence et d'un fort dynamisme interne, allait être reçu et développé dans la culture matérielle des siècles ultérieurs.

La second volet de la rencontre, consacré aux «constructions mémorielles", fut introduit par Nathania Girardin (Université de Neuchâtel: "Les objets de Charles: Charles le Chauve, ombre de Charlemagne?»), qui porta l'attention sur les nombreuses interventions de Charles le Chauve décelées par la critique historique, à propos des revendications médiévales d'objets liés à Charlemagne : cette collusion mémorielle doit sûrement aussi bien à l'homonymie des intéressés qu'à leurs politiques respectives, le plus jeune ayant cherché à réactualiser, par des dons d'objets fonctionnant comme une loupe temporelle, les initiatives de son grand-père. Philippe Cordez (Universität Hamburg: "L'histoire incarnée. Charlemagne et les reliques du Christ») étudiait ensuite le thème des reliques rapportées par Charlemagne de son voyage légendaire à Jérusalem, qui fut repris successivement en quelques décennies, à compter du milieu du XIe siècle, par plusieurs églises, de Saint-Denis à Aix-la-Chapelle et à Charroux. Charlemagne, fondateur prestigieux et modèle du croisé, y apparaît aussi comme le garant de l'authenticité des reliques revendiquées, ce qui fut décisif à Charroux où l'invention de la relique du prépuce s'inscrivit dans le contexte des débats sur la présence charnelle du Christ dans l'eucharistie: la figure profane de Charlemagne rejoint ici le point névralgique de la conception des objets mémoriels dans le christianisme. Cette association de Charlemagne aux reliques du Christ favorisa sûrement sa propre sacralisation, et finalement sa canonisation en 1164. Considérant son tombeau et ses reliquaires, Viola Belghaus (Université technique de Dresde : «Grab, Reliquien und Reliquiare Karls des Großen ») montra comment saint Charlemagne fut traité en tant qu'objet, mettant en lumière une tension entre la stratégie de ses successeurs sur le trône, désireux d'établir une relation privilégiée entre leur propre personne et ce modèle d'empereur chrétien, et celle des chanoines d'Aix-la-Chapelle, qui ne s'y refusaient pas mais ne procédèrent qu'avec prudence à l'institutionnalisation de ce culte atypique. 
Pierre Alain Mariaux (Université de Neuchâtel: «Topographies de la mémoire: les objets de Charlemagne ») rappela pour commencer que la notion d'« objet », toujours liée à celle de "sujet ", fut élaborée par les philosophes nominalistes du XIVe siècle et que sa projection sur les créations matérielles des siècles antérieurs constitue un obstacle à leur juste appréhension. Il invita ensuite à considérer d'une part les divers " objets de Charlemagne » (son sceptre, son cor, sa vaisselle, son jeu d'échecs, etc., qui existent généralement en plusieurs exemplaires) dans le contexte respectif de leur tradition littéraire, et d'autre part à étudier en chaque objet les stratégies plastiques de la matérialisation de ces « syntagmes ».

Synthétisant la discussion qui s'ensuivit, Anne-Orange Poilpré (Université de Nancy II), qui animait ce moment des débats, souligna la coexistence de différentes faces, développées au cours du temps, de la personnalité de "Charlemagne»: autant de portraits de "Charlemagne » en souverain chrétien, en fondateur d'églises, en saint empereur, en croisé, en familier des merveilles, matérialisés en autant de familles d'objets qui fondent des pans nouveaux de la culture matérielle médiévale. On conçut donc le projet de réunir ces objets dans un catalogue élaboré collectivement d'une manière aussi exhaustive que possible, organisé par types, et qui décrirait aussi bien leur identité matérielle que légendaire : son esquisse fait déjà apparaître la cohérence et l'intelligence plastique d'attributions dont on n'a longtemps su que se moquer. Établir ce "musée imaginaire» des objets légendaires d'un moment fondateur de l'occident médiéval est d'abord un travail d'érudition: toute suggestion est donc bienvenue. Il sera publié avec les actes du colloque, auxquels s'adjoindront encore plusieurs autres contributions.

$\mathrm{Au}$ terme de ces trois jours, la figure de "Charlemagne " s'est révélée comme un ensemble complexe de projections mémorielles superposées et éclatées, initiées par l'intéressé lui-même et déployées tout au long du Moyen Âge, et qui ne peuvent être approchées que par l'analyse archéologique de leurs manifestations textuelles et matérielles. La notion d'« objet ", quant à elle, est apparue comme un anachronisme, et comme un obstacle gênant nos efforts de compréhension au point qu'il nous est resté difficile d'éviter le mot! Mais la confrontation de "Charlemagne » et des "objets " a porté ses premiers fruits: de cette rencontre de Paris et d'Auxerre, on retiendra finalement que les «objets » construisant la mémoire, particulièrement nombreux aux XIIe et XIIIe siècles, ont un régime d'objectivité bien différent des «objets" carolingiens thésaurisant en eux le sacré et le politique, même s'ils sont en dernier ressort leur conséquence. Tous ces « objets » médiévaux trouvaient donc leur sens dans un régime transitif : ils étaient des « sujets » au sens moderne, qui n'ont sans doute pas fini de nous préoccuper.

\section{NOTES}

1. Lucas Burkart, Philippe Cordez, Pierre Alain Mariaux, Yann Potin (dir.), Le trésor au Moyen Âge. Discours, pratiques, objets, Florence : Sismel, à paraître (Micrologus' Library). 
2. Marie-Pierre Laffitte, Charlotte Denoël (dir.), Trésors carolingiens. Livres manuscrits de Charlemagne à Charles le Chauve, Paris : Bibliothèque Nationale de France, 2007. 